\title{
Complete Genome Report of Sphingobium Yanoikuyae S72. A Bacterium Capable of Degrading Hydrocarbons
}

Eric Sanchez-Lopez ( $\nabla$ ecoli_88@hotmail.com )

Centro de Biotecnologia Genomica, Instituto Politecnico Nacional

Temidayo Oluyomi Elufisan

Centro de Biotecnologia Genomica, Instituto Politecnico Nacional https://orcid.org/0000-0001-94450229

\section{Patricia Bustos}

Universidad Nacional Autonoma de Mexico Centro de Ciencias Genomicas

\section{Claudia Paola Charles-Mendoza}

Laboratorio Interaccion Planta-Microorganismos,Centro de Biotecnologia Genomica, Instituto

Politecnico Nacional

\section{Alberto Mendoza-Hererra}

Laboratorio interaccions Planta-Microrganismos, Centrro de Biotecnologia Genomica, Instituto

Politecnico Nacional

\section{Xianwu Guo}

Centro de Biotecnologia Genomica, Instituto Politecnico Nacional

\section{Research Article}

Keywords: Naphthalene, Phenanthrene, GC-MS, Biphenyl, Sphingobium, Toluene, genome, sequencing

Posted Date: July 22nd, 2021

DOl: https://doi.org/10.21203/rs.3.rs-687133/v1

License: (c) (i) This work is licensed under a Creative Commons Attribution 4.0 International License. Read Full License 


\section{Abstract}

Sphingobium yanoikuyae S72 was isolated from the rhizosphere of sorghum plant, Mexico. Its role on the degradation of polycyclic aromatic hydrocarbon $(\mathrm{PAH})$ was evaluated in a minimal medium containing one of biphenyl, naphthalene, phenanthrene, xylene and Toluene as the only carbon source. It grew effectively in each minimal medium (Bushnell Haas, BH). Gas column chromatography-mass spectrometry (GC-MS) analysis on the metabolite recovered after S72's growth in BH showed that it successfully degraded the PAHs tested resulting in the degradation of biphenyl (85\%), phenanthrene (93\%), naphthalene (81\%), xylene (19\%) and toluene (30\%). The complete genome of the S72 was sequenced to evaluate the genetic bases of S72 `s degradation of PAH. The genome was assembled de novo and reduced to 1 contig and consists of 5,532,623 bp, 5005 protein-coding genes. The analysis of the COG categories in S72 in comparison with other Sphingobium yanoikuyae and other PAH degrading gene, showed that it has in higher abundance in some categories that have been associated with the degradation of PAH (energy production and conversion (COG C, 6.35), carbohydrate transport and metabolism (COG G, 6.20\%), lipid transport and metabolism (COG I, 6.99), secondary metabolites biosynthesis (COG Q, 4.52\%), general function prediction only in transport and catabolism (COG R, 9.93),) Pan-core genome analysis revealed that about 126 unique genes in $\mathrm{S} 72$ are associated with the degradation of xenobiotics and PAHs. Most gene found in S72 are associated with the peripheral degradation pathway for PAH. Based on our observation we proposed a possible benzoate degradation pathway.

\section{Introduction}

Polycyclic Aromatic Hydrocarbon (PAH) are important pollutants which always find their way into the environment through various human activities such as the burning of fossil fuel, oil spillages and sometimes during vehicle repairs (Elufisan, 2020). The release of PAH to surrounding air is worrisome because of the associated hazards on people, animal and plants (United States National Academy of 1983). Exposures to fossil fuels and other PAH contaminants is known to disrupt agricultural lands, contaminate the surrounding air, and may induce cancer and other mutations in aquatic organisms, (White and Claxton 2004). The deleterious effects of the release of PAH to the environments make remediation processes inevitable. Several remediation processes have been employed for the cleaning of PAHs contaminated environment. Among such approaches are the use of chemicals and biological methods. Biological approaches have been identified as effective measures for the cleaning of sites contaminated by low molecular PAH (Gan et al. 2009). The degradation of high molecular weight PAH is said to be difficult because of their molecular structures. However certain bacteria have been reported to possess the capacity to degrade both high and low molecular weight PAHs. These bacteria have shown good potentials for the degradation of PAHs and could possibly be tools for modern bioremediation of PAH contaminated sites. Among such bacteria are member of the genus Stenotrophomonas, Pseudomonas, Franconibacter, Bacillus, Enterobacter among others (Juhasz and Naidu 2000; Kertesz* and Kawasaki 2010; Elufisan et al. 2020). Although there were arrays of bacteria that have been identified 
for the capacity to grow in and degrade $\mathrm{PAH}$, there are still paucity of information on the mechanisms being employed by many of them for the degradation of PAHs. Among such bacteria whose mechanism is yet to be fully elucidated are member of the genus Sphingomonads (Kertesz* and Kawasaki 2010).

The Sphingomonads belongs to the family Sphingomonadaceae. Yabuuchi et al. proposed the family Sphingomonadaceae in 1990 (Yabuuchi et al. 1990; 1998; Kosako et al. 2000). The family was later subdivided by Takeuchi et al. 2001 (Takeuchi et al. 2001; Euzéby 2004) into four genera:

Sphingomonas, Sphingobium, Novosphingobium, and Sphingopyxis. These genera are called the "sphingomonads." Members of the genera sphingomonads are commonly found in nature. There is an increased attention on these genera in recent times because they play important roles in the remediation of pollutants. They do so by using these pollutants as substrates for growth (Kim and Zylstra 1999; Cho et al. 2005; Cunliffe and Kertesz 2006; Ferraro et al. 2007; Gai et al. 2011). Among the sphingomonads with promising potential for both biodegradation and bioremediation of pollutants are the Sphingobium and Sphingomonas (Cunliffe and Kertesz 2006; Liu et al. 2012). Sphingobium species have been isolated and studied for their ability to degrade different pollutants including PAHs. However, most analysis on Sphingobium's capacity to degrade PAH were limited to the isolation of Sphingobium and evaluation of the metabolic products formed from PAH biodegradation.

Genome sequence analysis can unravel many underlining factors responsible for bacteria behavior. The needs to understand more about the biology of Sphigobium and its detail architecture with respect to PAH degradation necessitates a complete genome sequence analysis of a PAH degrading Sphingobium. In this study, we isolated Sphingobium yanokuyae S72 from the rhizosphere of sorghum sample collected from the northeastern part of Mexico (25.983333 latitude, 198.1 longitude, and altitude 120M a.s.l.). This strain grew effectively in PAH supplemented medium. Its potential to degrade PAH was further evaluated in a PAH degradation assay. The complete genome was sequenced and analyzed to understand the molecular mechanisms that are associated with its degradation of PAH.

\section{Materials And Methods}

\section{Bacterial isolation and characterization}

S72 was isolated from the rhizosphere of sorghum plant by a colleague in the lab in a previous study. One gram of soil sample recovered from sorghum plant rhizosphere was added to $10 \mathrm{ml} 0.85 \%$ saline solution in a $50 \mathrm{ml}$ falcon tube. The mixture was strongly agitated on a vortex and then serially diluted in sterile distilled water to the factor of $10^{-4} .0 .1 \mathrm{ml}$ of each diluent was inoculated on an already prepared Tryptic soy agar (TY) and incubated at $30^{\circ} \mathrm{c}$ for $24-48$ hours. The isolates which appeared on the plate were purified by subculturing individual colony in a freshly prepared TY agar and incubated in the same condition previously mentioned. The purified colonies which appeared on plates were identified morphologically with the electron microscope, biochemically using the API®20NE bacterial identification kit (bioMerieux, 200), and molecularly by amplifying the 16S rRNA fragment of the bacterial genome. The biochemical analysis was carried out using the API 20NE gallery system, which consists of a gallery 
made up of a series of microtubes with dehydrated medium and substrates. This system is used to identify non-enteric Gram-negative bacilli and some members of the Sphingomonas genus (bioMerieux sa, 2006). This test was carried out under the conditions specified by the manufacturer, (bioMerieuxsa). The galleries were incubated at $30^{\circ} \mathrm{C}$, and the results were observed at 24 and $48 \mathrm{~h}$. The interpretation of the positive and negative tests was made based on the API 20 gallery color chart (apiweb, bioMerieux, 2010). Subsequently, the results were compared with the API and ID 32 identification database (bioMeerieux) and with biochemical data assigned for Sphingobium yanoikuyae (Yabuuchi and Yoshimasa, 2002).

The genomic DNA for the bacterial identification was extracted from an overnight broth culture of the suspected colonies with the wizard Promega genomic DNA extraction kit (USA) according to the manufacturer's instruction. The extracted genomic DNA was amplified with the bacterial universal primer $27 f$ ( $5^{\prime}$-GAGAGTTTGATCCTGGCTCAG -3') and 1495r (5' - CTACGGCTACCTTGTTACGA-3') (Grifoni et al. 1995). The amplified region was sequenced and blast check analysis on the NCBI database was carried out to determine the identity of the bacteria.

2. Bacterial growth in, and degradation of PAHs

S. yanoikuyae $\mathrm{S72}$ was inoculated in different Bushnell Haas $(\mathrm{BH})$ agar media, which were already supplemented with PAH at a final concentration of $0,20,40,80100$ and $150 \mu \mathrm{g} / \mathrm{mL}$, respectively. The final bacterial concentration inoculate in the medium was approximately $3.0 \times 10^{7} \mathrm{CFU} / \mathrm{ml}$. Each culture was incubated at $30^{\circ} \mathrm{C}$ for 72 hours. The highest concentration of PAH tolerated by the isolates were selected for the degradation studies.

The PAHs-degrading test was carried out in $5 \mathrm{BH}$ liquid media, with each containing one out of the 5 PAHs (naphthalene, phenanthrene, biphenyl, toluene, or xylene) as the sole carbon source. A $1 \mathrm{ml}$ of $S$. yanoikuyae 572 was added to $40 \mathrm{ml}$ of 5 Bushnell Haas Broth (BH) already supplemented with each of the PAHs, in a $250 \mathrm{ml}$ flask, respectively. The culture was then incubated in a rotatory incubator with revolutions of $180 \mathrm{rpm}$ at $30^{\circ} \mathrm{C}$ for 25 days. The bacterial growth was checked every 48 hours and growth was determined using the spectrophotometer. The cultures were withdrawn on the 12th and 25th day to determine the extent of PAHs degradation by the isolate. The metabolites formed were determined by a Gas Column chromatography mass spectrophotometry (GC-MS) analysis.

\section{GC-MS chromatography analysis}

For the GC-MS analysis, an Agilent Technologies brand gas chromatograph, model 6890N (Net Work GC system), equipped with a DB-5 column, 5\% -phenyl-methyl-polysiloxane (Agilent Technologies), 60 meters long, $0.25 \mathrm{~mm}$ in internal diameter and $0.25 \mu \mathrm{m}$ thick film was used. The starting temperature was $50^{\circ} \mathrm{C}$, which was maintained for $5 \mathrm{~min}$, subsequently the temperature was raised to $280^{\circ} \mathrm{C}$ using a heating ramp of $20^{\circ} \mathrm{C} / \mathrm{min}$, for $20 \mathrm{~min}$. Helium was used as carrier gas at a flow of $1 \mathrm{~mL} / \mathrm{min}$, the injector temperature was $250^{\circ} \mathrm{C}$, split injection, with a split ratio of 50: 1 . Once the chromatogram was obtained, the identification of each of the peaks was carried out by mass spectrometry using an Agilent 
Technologies model 5975 inert XL mass spectrometer. Mass spectra were obtained by electron impact ionization at $70 \mathrm{eV}$, for identification the mass spectra obtained for each compound were compared with a database (HP Chemstation-NIST 05 Mass Spectral search program, version 2.0d), in addition to the comparison with a standard, analyzed under the same conditions, which was used as an external standard for quantification.

The GC-MS analysis started with the separation of the organic phase and the aqueous phase of the culture medium in a rotatory funnel. The culture medium $(40 \mathrm{ml})$ was poured in a separating funnel in which $20 \mathrm{~mL}$ of HPLC grade hexane (J.T. Baker ${ }^{\circledR}$ ) were added. The funnels were manually shaken for 10 min. Funnels were kept at rest until the two phases were clearly separated into two. From the upper phase containing the $\mathrm{PAH}, 1 \mathrm{~mL}$ aliquot was transferred to $1.5 \mathrm{~mL}$ dark vials. The was used for the analysis of the metabolite formed in a gas chromatograph (Agilent Technologies ${ }^{\circledR}$ Model $6890 \mathrm{~N}$ ). Once the chromatograms were obtained, each of the chromatographic peaks was identified by mass spectrometry, using a mass detector (Agilent Technologies ${ }^{\circledR}$ Mod. 5975 inert XL). The mass spectra observed in the metabolites were confirmed with the HP Chemstation-NIST 05 Mass Spectral Search Program, version $2.0 \mathrm{~d}$ software. The spectra were compared with the standard for each $\mathrm{PAH}$ and the spectra from the control in which no bacterium was inoculated under the same condition. Experiment was set, in which treatments consisted of flasks inoculated with or without $S$. yanoikuyae, and with or without PAH. Each type of PAH was separately analyzed, and each treatment had five replicates in the experiment. The experiments were repeated three times. Data were analyzed using the SAS statistical program (Dunne 2005).

\section{Genome sequencing and analysis of $S$. yanoikuyae $S 72$.}

We sequenced the genome of S72 to fully understand its genetic basis for the degradation of PAH.

The genomic DNA was extracted with the Promega ${ }^{\circledR}$ Wizard Genomic DNA purification kit (Promega Corp., Madison, WI, USA) according to manufacturer's recommended protocol. The whole genome sequencing was performed using the Illumina MiSeq ${ }^{\text {TM }}$ platform (Illumina, Inc., San Diego, CA, USA). The reads obtained from the genome assembly were trimmed with trim-galore, which also check the quality of the read with its embedded fastqc. The genome assembly was carried out in 2 stages: i) de novo assembly with the Spades Genome Assembler (Bankevich et al. 2012) and Velvet (Zerbino and Birney 2008) and ii). reference-based assembly.

The de novo assembly used two different bacterial genome assembly pipelines, which are the Spades based de novo assembly (3.9.1) and the velvet assembly pipeline. The assembled genome from the two software were compared using the Metassembler software. A consensus assembly produced from the two assembling techniques was then used for the reference-based assembly (Wences and Schatz 2015). The reference-based assembly was carried out to reduce the genome to 1 contigs. This approach employs the alignment of the consensus assembly with Sphingomonas genome (ATCC512030) recovered from NCBI database using the Nucmer software and the Consed suite (Gordon and Green 2013; Marçais et al. 2018). The assembled genome was annotated with Prokka (version 1.12) and NCBI 
prokaryotic genome annotation pipeline were used for automatic gene prediction and annotation (Seemann 2014; Tatusova et al. 2016). RNAmmer (Lagesen et al. 2007) and tRNAscan (Lowe and Eddy 1996) were used for the identification of rRNA and tRNA. The KEGG and Orthologous gene cluster analysis was carried out on the web based PATRIC server (Wattam et al. 2014). Alternatively, COGs for unique genes were assigned with BlastP against the COGs database downloaded from NCBI. The bacterial pan genome analysis (BPGA) tool was used for the analysis of the isolate's pan genome (Chaudhari et al. 2016). The Average Nucleotide Identity based on Mummer (ANIm) was determined with PYANI (Pritchard et al. 2016). The genomic island in S72 was identified with an online web-based Island viewer4 (Bertelli et al. 2017) and pairwise analysis on Nucmer (Marçais et al. 2018). The deep gene sequence analysis for the identification of the genes that were associated with the degradation of PAH and comparative genomics such as COG abundance was carried out on the JGIIMG database (Mavromatis et al. 2009).

\section{Results}

S72 is a gram-negative rod and moves by means of a monotrichous flagellum. It is between 1.0-3.5 $\mu \mathrm{m}$ in length and 0.6-1 $\mu \mathrm{m}$ in width (Fig. S1). S72 effectively use D glucose, $L$ arabinose, Mannose, $N$ acetyl glucosamine, maltose, potassium gluconate, Malic acid, citrate, galactose, citrate and esculin for its growth. It however could not use mannitol, adipic acid, capric acid, phenyl acetic acid as a substrate for its growth (Table 1).

The preliminaries analysis showed that $\mathrm{S} 72$ can grow in the minimal media $(\mathrm{BH})$ supplemented with polycyclic aromatic hydrocarbons (PAH) (biphenyl, phenanthrene, xylene, toluene, and naphthalene). The number of colonies forming units appearing on the plates decreased as the concentration $(0,20,40,80$ 100 and $150 \mu \mathrm{g} / \mathrm{mL}$ ) of the PAH in the BH medium increased. The preliminary analysis also revealed that the $S 72$ could better tolerate the hydrocarbons at a concentration that is $\leq 100 \mu \mathrm{g} / \mathrm{ml}$, but their growth in PAH began to decline at any concentration greater than $100 \mu \mathrm{g} / \mathrm{ml}$. S72 did not show any growth when inoculated in $\mathrm{BH}$ medium containing $150 \mu \mathrm{g} / \mathrm{ml}$ toluene or xylene and showed limited growth in plate with naphthalene, phenanthrene or biphenyl at this concentration. Also, the rate at which $\mathrm{S} 72$ grew in medium containing toluene and xylene is lower than other PAHs tested at $100 \mu \mathrm{g} / \mathrm{ml}$. (Fig. 1). Based on the reported observations $100 \mu \mathrm{g} / \mathrm{ml}$ was selected as the study concentration for the experiment.

S72 degraded all the PAHs tested at $100 \mu \mathrm{g} / \mathrm{ml}$ with varying efficiencies. S72 showed the best activity in phenanthrene, resulting in the $93 \%$ degradation. The degradation of biphenyl, and naphthalene lead to 85 and $81 \%$ reduction, respectively (Fig. 2). However, a low degradation efficiency was observed in the medium containing xylene (19\% and $31 \%$ ) respectively. The degradation was confirmed by the reduction in the peak for the PAHs as compared to the control from the GC-MS analysis (Fig 3, S2).

Genomic analysis has been used severally to unravel several details about bacterial behavior. As a result of this, the genome of $S 72$ was sequenced and analyzed to relate its function with its genetic constituent. The sequenced genome of $S$. yanoikuyae $S 72$ was reduced to 1 contig, consisting of 5,532,633 bp, 5005 
CDS, and 4 identical copies of the rRNA gene operon (23S, $16 \mathrm{~S}$ and $5 \mathrm{~S})$. The overall $\mathrm{G}+\mathrm{C}$ content of the assembled genome is $64.23 \%$. The genome has 5231 putative genes and 75 RNAs (67tRNA and 12 rRNA). 1140 genes are in operons, 2515 are parts of the clusters of orthologous gene (COGs) Table2. S72 has some COG categories in abundance than some other Sphingobium species which has been previously reported. These COG categories include energy production and conversion (COG C, 6.35), carbohydrate transport and metabolism (COG G, 6.20\%), lipid transport and metabolism (COG I, 6.99), secondary metabolites biosynthesis (COG Q, 4.52\%), general function prediction only in transport and catabolism (COG R, 9.93) (Table 3), In a related analysis S72 was found to have higher COG abundance than 17 other hydrocarbon degrading bacteria retrieved from IMG database Table 4). It showed abundance in the COG categories Mobilome: prophages, transposons (COG X, 2.03\%), Secondary metabolites biosynthesis, transport and catabolism (COG Q, 4.52\%), Carbohydrate transport and metabolism (COG G, 6.20\%), Lipid transport and metabolism (COG I, 6.99\%), Cell wall/membrane/envelope biogenesis (COG M, 6.58), Replication, recombination and repair (COG L, $3.33 \%$ ), Inorganic ion transport and metabolism (COG P, 6.22\%), Transcription (COG K, 7.8), Posttranslational modification, protein turnover, chaperones ( COG, 0, 4.27\%), and General function prediction only (COG R, 9. 93\%) Some of these COG categories have been previously reported to be associated with the degradation and mineralization of PAHs (Pal et al. 2017; Elufisan et al. 2019). The deep sequence analysis of S72 showed that it possesses 37 genes in different COG categories which are associated with the degradation of xenobiotics and polycyclic aromatic hydrocarbons. These genes include 2 pyrone-4 - 6 - decarboxylase hydrolase (A6768_17510, COG R), 4 carboxy

- 2 - hydroxy muconic semialdehyde dehydrogenase (A6768_17470, COG R). These two genes have been linked to the degradation of Benzoate and Fluorobenzoate by bacteria. Bacteria have been reported to use the enzyme encoded by these genes for the degradation of benzoate via hydroxylation (Oltmanns et al. 1989). Others include S-(hydroxymethyl) glutathione dehydrogenase/alcohol dehydrogenase (A6768_05785, COG R) that is associated with the degradation of naphthalene and chloroalkane (Yang et al. 2017). S-(hydroxymethyl) glutathione dehydrogenase/alcohol dehydrogenase has been previously reported to be associated with the degradation of naphthalene (Das et al. 2015; Pal et al. 2017). Thus, the presence of S-(hydroxymethyl) glutathione dehydrogenase/alcohol dehydrogenase in S72 could be associated with its need to degrade naphthalene as a substrate for growth. Alcohol dehydrogenase (cytochrome c) (A6768_00755, COG G), aldehyde dehydrogenase (NAD+) (A6768_10925, COG E), aldo/keto oxidoreductase (A6768_11370, COG R), Phenylacetaldehyde dehydrogenase (A6768_11850, COG C) known to participate in the degradation of fluorobenzoate toluene and naphthalene are also present in S72 ( $\mathrm{Pal}$ et al. 2017; Elufisan et al. 2020). Other PAH degrading genes identified in S72 include propanol-preferring alcohol dehydrogenase (A6768_17370, EC:1.1.1.1), carboxymethylenebutenolidase (A6768_23915), catechol 1,2-dioxygenase (A6768_16795), muconate cycloisomerase (A6768_16805), aryl-alcohol dehydrogenase (A6768_11850), 2-keto-4-pentenoate hydratase (A6768_14055), oxalocrotonate tautomerase (A6768_04785) benzoate 1,2-dioxygenase alpha subunit (A6768_16790), benzoate/toluate 1,2-dioxygenase beta subunit (Ben B), dihydroxy cyclohexadiene carboxylate dehydrogenase (Ben D). Similarly, a gene encoding the lactoylglutathione lyase was found in S72. The 
lactoylglutathione lyase enzyme has been described to be actively involved in the cleavage of aromatic bond in many aromatic hydrocarbons (Mesarch et al. 2000). Among the observed genes in $S 72$ are genes which have been reported to be specifically associated with the degradation of toluene. The genes include 4 genes encoding 3-hydroxyacyl-CoA dehydrogenase (EC:1.1.1.35) one gene for aryl-alcohol dehydrogenase (EC:1.1.1.90), and a gene for Catechol 1,2-dioxygenase (EC:1.13.11.1). Others include 2 genes encoding the enzyme oxidoreductases (EC:1.14.13.-) which often act on paired donors, with incorporation or reduction of molecular oxygen. 3 copies of the genes encoding Ferredoxin-NAD (+) reductase (EC:1.18.1.3), 7 copies of Acyltransferases (EC:2.3.1), 3 copies of carboxymethylenebutenolidase and 1 copy of muconate cycloisomerase.

S72 relatedness to other Sphingobium strain (105) was evaluated on PYANI (Pritchard et al. 2016) using the average nucleotide identity mummer (ANIm) comparison measure. The ANIm result showed that S72 is closely related to S. yanoikuyae strain UBA2097 sharing $97 \%$ average identity with it (Fig. 4). The analysis of $S 72$ pan genome showed that it shared 1734 core genome with other Sphingobium species and possess 403 unique genes. We noted that 126 of the unique genes are associated with the catabolism of xenobiotics. Twenty out of the unique genes have been previously reported to be involved in the degradation of toluene, xylene, ethylbenzene, biphenyl, benzoate, naphthalene, anthracene, tetrachloroethene, 1, 4-dichlorobenzene, bisphenol, trinitrotoluene in bacteria. These genes are involved in both the catabolism of central aromatic intermediate and the peripheral catabolic pathway for aromatic hydrocarbon. (Table S1).

Horizontal gene transfer is a common phenomenon through which bacteria often acquire some genes that are essential for their survival (Elufisan et al. 2020). The acquired genes are commonly found on the genomic islands in bacteria or as mobile genetic elements. The analysis of the S72's genome with the online based Island viewer 4.0, revealed that it has 37 genomic islands (Fig. 5). Five large regions were found in the genomic Island with regions II and III being the largest consisting of 317,308 kb and 579,060 $\mathrm{kb}$ in length, respectively. An in-depth look into the genomic island showed the presence of many genes that are associated with the degradation of xenobiotics and PAHs. Among such genes are SDR family NAD(P)-dependent oxidoreductase (A6768_07840), aldo/keto reductase (A6768_07825), cytochrome P450 (A6768_11830), 4-hydroxybenzoate 3-monooxygenase (A6768_12975), and aromatic alcohol reductase (A6768_RS13080). The other PAH degrading genes found on the genomic island can be seen in supplementary file.

\section{Discussion}

The biochemical characteristics and the molecular identification of $S 72$ confirmed that it is a $S$. yanoikuyae strain. It grew effectively in the tested PAH concentration until $100 \mathrm{microgram} / \mathrm{ml}$ implying that $\mathrm{S} 72$ can use the tested PAH as substrates for growth. S72's growth rate, however, decreased as the PAH's concentration increased. The reduction in growth as shown in Fig. 1 could be due to increased toxicity that is associated with increased in the PAH concentration. This toxicity may be due to the reduction in rate of solubility that may occur as PAH concentration increases. Previous study has shown 
that as concentration of hydrocarbon increases the rate of solubility decreases resulting in the reduction in growth for bacteria (Volkering et al. 1992). Low solubility could make it difficult for bacteria to utilize certain compounds as nutrient for growth (Leahy and Colwell 1990).

S72 successfully degraded the PAHs during the 25-days degradation study. S72 showed the most activities in the medium containing phenanthrene. The activity of S72 resulted in the degradation of Phenanthrene by $93 \%$ at a concentration of 100 microgram $/ \mathrm{mL}$. This observation is evident by the reduction in the spectra for Phenanthrene following the activity of S72 in contrast to the control which lack bacteria but only PAH (Fig. 3). This observation is like a previous report in which Sphingobium chlorophenolicum C3R degraded Phenanthrene by 65\% (Liu et al. 2019). Hence, S72 possess the capability to degrade Phenanthrene but has a lower efficacy compared to Sphingobium chlorophenolicum C3R because it cannot degrade the PAHs at a conc greater than $100 \mu \mathrm{l} / \mathrm{mL}$. S72's ability to degrade PAH is not limited to Phenanthrene (PHE), it also degraded biphenyl and naphthalene by 85 and $81 \%$ respectively. This may imply that $\mathrm{S} 72$ had learnt to survive in and use different PAH as substrates for growth. S72 degradation efficiency is slightly weaker than what was reported for Burkholderia fungorum FM-2 which degraded PHE by $65 \%$ after 3 days (Liu et al. 2019). It however has an advantage of surviving in PHE and ensuring its degradation after 25days. On the other hand, S72 did not effectively degrade xylene and toluene. Several reason could be responsible for the inability of S72 to degrade toluene and xylene, one of such reason could be the presence of a $\mathrm{CH} 3$ and two $\mathrm{CH} 3$ groups as an attachment to their benzene rings, respectively. It may be difficult for $\mathrm{S} 72$ to degrade the single benzene ring in xylene and toluene unlike in the other PAHs with more than one benzene ring and other moieties. In addition, the tested concentrations are not the molar equivalent but weight concentration. The same weight concentration in the media may contain more molecules of xylene and toluene than in the other PAHs, which may also increase toxicity of these compounds (higher molar concentration) on S72. Previous studies have shown that high concentration can inhibit the ability of bacteria to degrade PAH (Leahy and Colwell 1990). Also, the in-depth analysis of the genome of S72 showed that the genes encoding the enzymes required for the degradation of toluene are not complete in it and such may be another reason for its inability to degrade toluene. It has only four genes that that are directly associated with the degradation of toluene, but these genes are taking part in different reactions in the pathway for the degradation of toluene. The genes are aryl-alcohol dehydrogenase [EC:1.1.1.90], an Oxidoreductases acting on the $\mathrm{CH}-\mathrm{OH}$ group of donors with $\mathrm{NAD}+$ or NADP+ as acceptor, muconate cycloisomerase [EC:5.5.1.1] a lyase which isomerizes 3 methyl cis cis muconate to 3 methyl muconolactone. The other two genes encode catechol 1, 2 dioxygenase and carboxymethylenebutenolidase [EC:3.1.1.45]. Catechol 1, 2 dioxygenase catalyzes the decyclizing of the aromatic ring of methyl catechol to generate 3-methylcis, cis-hexadienedioate, while carboxymethylenebutenolidase [EC:3.1.1.45] is acting as dienelactone hydrolase converting 3-chloro-2-methyldienelactone to 3-Chloro-2-methylmaleylacetate in the breakdown of toluene. This observation is same for the genes required for the degradation of xylene as reported by the KEGG database.

S72's ability to grow in and degrade PAHs has underlying molecular mechanisms. The molecular mechanisms responsible for the survival of S72 in and degradation of PAHs was evaluated by the 
sequencing of its genome. The genomic analysis of the $\mathrm{S} 72$ genome show the presence of some genes which are essential for the degradation of PAHs. Genes such as S-(hydroxymethyl) glutathione dehydrogenase/alcohol dehydrogenase (A6768_05785, COG R) which is known to enhance the degradation of PAH by catalyzing the oxidation of long chain primary alcohol (Uniprot). Alcohol dehydrogenase which are essential for the degradation of PAH via their oxido-reductase activities. We also noted several copies of other essential genes for the degradation of PAH. For example, there were 18 genes that are associated with the Cytochrome P450 superfamily. The cytochrome P450 is a monooxygenase which are involved in the degradation of PAH. Recent report showed that cytochrome P450 can play important role in the degradation of naphthalene and pyrene (England et al. 1998). The presence of cytochrome P450 monooxygenase (CP450) in S72 may be due to its role in helping S72 in the degradation of PAHs. CYP450's importance in S72 is evident with the presence of many of its copies in S72. 18 genes which are member of CYP450 superfamily were found in S72. The role of the unique CYP450 gene could not be fully explained here but it is likely that they are involved in some pathways that are associated with the degradation of PAH.

Similarly, several genes which have been linked with the degradation of tetrachloroethane were seen in the genome of S72. These genes include ntrilotriacetate monooxygenase (A6768_04895, EC 1.14.13.), Aldehyde dehydrogenase (A6768_04870, EC 1.2.1.3) and Alcohol dehydrogenase (A6768_22375, EC 1.1.1.1). There are 22 genes in these three categories that have been associated with the degradation of tetrachloroethane. Tetrachloroethane ( $\mathrm{CCl} 4)$ and trichloroethanes are organic solvents commonly used for laundries and metal cleaning, as a result they are often find as environmental pollutant (Ryoo et al. 2001). No aerobic bacteria have been reported to possess the ability to degrade $\mathrm{CCl} 4$ (Gerritse et al. 1997), The presence of genes needed to degrade CCl4 may suggest that $S 72$ possess the tendencies to decontaminate an area polluted by $\mathrm{CCl} 4$. it a potential bacterium for the aerobic decontamination of $\mathrm{CCl} 4$ polluted sites. S72's ability to grow in and degrade naphthalene is associated with the presence of some genes in its genome.

The genome has six genes which are involved in the pathway for the degradation of naphthalene (Alcohol dehydrogenase, Aldehyde dehydrogenase, and Nitrilotriacetate monooxygenase). Although these genes are not the only one that are needed in the pathway for the degradation of naphthalene, their presence in S72's genome may be the reason for its naphthalene degradation. More so, there are several hypothetical genes in S72 which are in the same operon with some of the genes that are involved in the degradation of PAHs (Fig. S3) and, as such may be complementing the roles of the identified genes in the degradation of naphthalene (Fig. S3). For example, we noted two genes encoding hypothetical protein upstream to the gene encoding nitronate monooxygenase and one hypothetical protein gene downstream nitronate monooxygenase. Most genes in this region had been previously reported to play essential role in the adaptation to PAH environment, uptake of $\mathrm{PAH}$, utilization and degradation of PAH. They include the lysR family protein gene which is known to play significant role in the regulation of bacterial adaptation to stress and the use of PAH. 
Comparative genomic analysis of S72 showed that it is more closely related to $\mathrm{S}$. yanoikuyae strain UBA2097 with which it shares $97 \%$ average nucleotide identity confirming it a Sphingobium yanoikuyae strain. Further analysis on its genome showed that it has higher abundance in some cog categories (Mobilome: prophages, transposons (COG X), Secondary metabolites biosynthesis, transport and catabolism (COG Q), Carbohydrate transport and metabolism (COG G), Lipid transport and metabolism (COG I), Cell wall/membrane/envelope biogenesis (COG M), Replication, recombination and repair (COG L), Inorganic ion transport and metabolism (COG P), Transcription (COG K), Posttranslational modification, protein turnover, chaperones ( $C O G, 0$ ), and General function prediction only (COG R)) when compared with previously described PAH degrading bacteria retrieved from JGI_IMG database. Many enzyme that are essential for the degradation of PAH by bacteria have been reported to be encoded by genes in some of these COG categories (Das et al. 2015). For example, it has a higher abundance of the COG category $\mathrm{K}$ which is the transcription category. There are 307 genes in this cog category, 49 of which belong to the LTTR family. The LTTR has been described as regulator essential for the survival of bacteria in diverse environment. Pal et al 2017, (Pal et al. 2017) reported that the abundance of the LTTR family in Franconibacter could be associated with their utilization of PAH Binnewies et al., had previously reported that the LTTR family plays important role in regulating genes responsible for aromatic compound catabolism, motility of cell and quorum sensing (Binnewies et al. 2006). The abundance of the LTTR gene family in S72 implies that it highly metabolically active and thus could be associated with its capacity to degrade PAHs. Twenty-one proteins of the OmpR family transcriptional regulator of the two components system, 6 Mer family regulator protein, 21 proteins belonged to the multiple antibiotic resistance regulator (MarR) family (COG1846) (Wilkinson and Grove 2006); S72 has 41 TetR regulation protein (COG1309 and COG3226), and 14 GntR family transcriptional regulators protein (COG1167, COG1802, COG2186 and COG2188). These transcription regulators are known to play essential roles in the degradation of aromatic hydrocarbon (Gerischer 2002). Other transcriptional regulating protein in S72 include 23 AraC family (COG, 2207, COG4977, COG1609) known to be involved in the metabolism of different sugars (Martin and Rosner 2001), 8 proteins of the IcIR family transcriptional regulator (COG1414), 2 proteins in the AsnC transcriptional regulator and 8 proteins of the ArsR family transcriptional regulator. $\mathrm{S} 72$ also has gene in the two-component system which are responding to the limitation of phosphate in the environment. It has a Pho $\mathrm{R}$ and Pho $\mathrm{B}$ genes which are phosphate regulon regulator in the $\mathrm{OmpR}$ family which can play essential role in the phosphate transport system (Pal et al. 2017).

S72 also has in abundance genes in the COG category for Mobilome: prophages, transposons (COG X), This COG category comprise genes that were acquired via horizontal genes transfer, some studies have shown that bacterial adaptation to polycyclic aromatic hydrocarbon could be mediated by an acquired mobile genetic element such as transposons, integron, plasmids or even prophages (Top and Springael 2003). There are about 80 genes in this COG category. The high abundance of gene from the COG $X$ category could be an indication that certain genes which are essential for the degradation of PAH might have be a part of the mobilome, prophage or transposon.

\section{Conclusions}


S. yanoikuyae S72 was isolated from northern Mexico. S72 used selected PAHs as unique carbon source for its growth. GC-MS analysis showed that S72 effectively degraded biphenyl, phenanthrene, and naphthalene. Genome analysis revealed the genes responsible for degradation of the tested PAH in $S$. yanoikuyae S72 genome. Most genes found in S72 are associated with the peripheral pathway for the degradation of the tested PAH. Although, some genes that are associated with the degradation of PAH were not found in S72, there were many hypothetical genes in S72 in the same region with the genes that are involved in the degradation of PAHs. These hypothetical genes may have roles in the degradation of the tested PAH as observed in the experimental study. S. yanoikuyae S72 strain has a potential to be used as an agent for cleaning up environments contaminated with petroleum and other chemical pollutants. However, the use of microorganisms for bioremediation are highly dependent on other abiotic and biotic soil parameters. Thus, necessitating further "in situ" studies to investigate its activity and ability to survive in hydrocarbons contaminated soils would be essential.

\section{Nucleotide sequence accession number}

The complete genome sequence of $S$. yanoikuyae 372 was deposited at GenBank with accession number $\mathrm{CP} 023741$. This strain is deposited at laboratory interaction environment-microorganism culture collection center.

\section{Declarations}

\section{Declaration}

Ethical approval: The study does not involve human or animal and so does not require any ethical approval.

Consent for publication: No individual data was used in the study and so does not require any consent before sending out for publication.

Competing interest: There are no financial or Nonfinancial competing interest on the manuscript.

Conflict of interest: Temidayo O Elufisan, Patricia, Bustos, Paola Charles-Mendosa, Alberto Hererra Mendosa and Xianwu declare that they do not have conflict of interest.

Funding: There is no special funding for this study.

\section{Authors' contribution}

Author Claudia Paola, Charles-Mendoza and Eric Sanchez Lopez designed and carried out the experimental study.

Author Eric Sanchez-Lopez wrote the manuscript.

Author Xianwu Guo and Author Alberto Hererra Mendoza supervised the study. 
Author Patricia Bustos carried out the genome assembly and analysis.

Author Temidayo O Elufisan edited the manuscript in English.

\section{Authors Information}

Author Claudia Paola is professor in UMAN university, where she currently works in the clinical laboratory of UMAN University. The line of knowledge of it is the isolation of microorganisms present in the rhizosphere of the soil.

Author Eric Sanchez-Lopez is a candidate for a doctorate in genomic biotechnology, is awarded a scholarship by the national council of science and technology of Mexico. He currently works in the Academic Technical Directorate. The research line is focused on the search for microorganisms with biotechnological potential.

Author Xianwu Guo is a professor at the Centro de Biotecnologia Genomica, Instituto Politecnico Nacional, Mexico where he currently works on the molecular genomics of bacterial behavior and their interaction with the environment. He is working on elucidating the mechanism involved in prey susceptibility to bdellovibrio. He is currently the head of Genomic Biotechnology laboratory of the Centro de Biotecnologia Genomica, Instituto Politecnico Nacional Mexico.

Author Patricia Bustos works at the Center for Genomic Sciences of the Autonomous University of Mexico, she is currently attached to the research group in the Evolutionary Genomics Program. Her work has focused on the area of Microbial Genomics, being a highly specialized technique in automated sequencing, assembly, bioinformatic analysis and sequence annotation methodologies.

Author Temidayo $\mathrm{O}$ Elufisan holds a $\mathrm{PhD}$ in biotechnology with a focus on bacteria genomics. He has previously worked on the Isolation and Genomic characterization of Stenotrophomonas from the environment. He also worked on the application of Stenotrophomonas species on the degradation of Polycyclic Aromatic Hydrocarbon. He is currently working on the purification of town water supply using moringa seeds.

\section{Acknowledgements}

To Lourdes Vital, Ma. Antonia Cruz and Susana Fernandez for technical assistance and suggestion to improve the genome analysis.

\section{References}

1. Bankevich A, Nurk S, Antipov D, et al (2012) SPAdes: a new genome assembly algorithm and its applications to single-cell sequencing. J Comput Biol 19:455-77.

https://doi.org/10.1089/cmb.2012.0021 
2. Bertelli C, Laird MR, Williams KP, et al (2017) IslandViewer 4: Expanded prediction of genomic islands for larger-scale datasets. Nucleic Acids Res 45:W30-W35. https://doi.org/10.1093/nar/gkx343

3. Binnewies TT, Motro Y, Hallin PF, et al (2006) Ten years of bacterial genome sequencing:

4. Comparative-genomics-based discoveries. Funct. Integr. Genomics 6:165-185

5. Chaudhari NM, Gupta VK, Dutta C (2016) BPGA-an ultra-fast pan-genome analysis pipeline. Sci Rep 6:. https://doi.org/10.1038/srep24373

6. Cho O, Choi KY, Zylstra GJ, et al (2005) Catabolic role of a three-component salicylate oxygenase from Sphingomonas yanoikuyae B1 in polycyclic aromatic hydrocarbon degradation. Biochem Biophys Res Commun 327:656-662. https://doi.org/10.1016/j.bbrc.2004.12.060

7. Cunliffe M, Kertesz MA (2006) Effect of Sphingobium yanoikuyae B1 inoculation on bacterial community dynamics and polycyclic aromatic hydrocarbon degradation in aged and freshly $\mathrm{PAH}-$ contaminated soils. Environ Pollut 144:228-237. https://doi.org/10.1016/j.envpol.2005.12.026

8. Das D, Baruah R, Sarma Roy A, et al (2015) Complete genome sequence analysis of Pseudomonas aeruginosa N002 reveals its genetic adaptation for crude oil degradation. Genomics 105:182-190. https://doi.org/10.1016/j.ygeno.2014.12.006

9. Dunne A (2005) Introduction to Categorical Data Analysis. Popul Anal Gr Eur 14

10. Elufisan TO, Lozano L, Bustos P, et al (2019) Complete Genome Sequence of Stenotrophomonas maltophilia Strain SVIA2, Isolated from Crude Oil-Contaminated Soil in Tabasco, Mexico. Microbiol Resour Announc 8:. https://doi.org/10.1128/mra.00529-19

11. Elufisan TO, Rodríguez-Luna IC, Oyedara OO, et al (2020) The Polycyclic Aromatic Hydrocarbon (PAH) degradation activities and genome analysis of a novel strain Stenotrophomonas sp. Pemsol isolated from Mexico. PeerJ 8:e8102. https://doi.org/10.7717/peerj.8102

12. England PA, Harford-Cross CF, Stevenson JA, et al (1998) The oxidation of naphthalene and pyrene by cytochrome P450(cam). FEBS Lett 424:271-274. https://doi.org/10.1016/S0014-5793(98)001896

13. Euzéby J (2004) Validation of publication of new names and new combinations previously effectively published outside the IJSEM. Int J Syst Evol Microbiol 54:1909-1910. https://doi.org/10.1099/ijs.0.63512-0

14. Ferraro DJ, Brown EN, Yu CL, et al (2007) Structural investigations of the ferredoxin and terminal oxygenase components of the biphenyl 2,3-dioxygenase from Sphingobium yanoikuyae B1. BMC Struct Biol 7:. https://doi.org/10.1186/1472-6807-7-10

15. Gai Z, Wang X, Tang H, et al (2011) Genome sequence of Sphingobium yanoikuyae XLDN2-5, an efficient carbazole-degrading strain. J. Bacteriol. 193:6404-6405

16. Gan S, Lau E V., Ng HK (2009) Remediation of soils contaminated with polycyclic aromatic hydrocarbons (PAHs). J. Hazard. Mater. 172:532-549

17. Gerischer $U$ (2002) Specific and global regulation of genes associated with the degradation of aromatic compounds in bacteria. J Mol Microbiol Biotechnol 4:111-121 
18. Gerritse J, Kloetstra G, Borger A, et al (1997) Complete degradation of tetrachloroethene in coupled anoxic and oxic chemostats. Appl Microbiol Biotechnol 48:553-562. https://doi.org/10.1007/s002530051096

19. Gordon D, Green P (2013) Consed: A graphical editor for next-generation sequencing. Bioinformatics 29:2936-2937. https://doi.org/10.1093/bioinformatics/btt515

20. Grifoni A, Bazzicalupo M, Di Serio C, et al (1995) Identification of Azospirillum strains by restriction fragment length polymorphism of the 16S rDNA and of the histidine operon. FEMS Microbiol Lett 127:. https://doi.org/10.1016/0378-1097(95)00042-4

21. Juhasz AL, Naidu R (2000) Bioremediation of high molecular weight polycyclic aromatic hydrocarbons: A review of the microbial degradation of benzo[a]pyrene. Int. Biodeterior. Biodegrad. 45:57-88

22. Kertesz* MA, Kawasaki A (2010) Hydrocarbon-Degrading Sphingomonads: Sphingomonas, Sphingobium, Novosphingobium, and Sphingopyxis. In: Handbook of Hydrocarbon and Lipid Microbiology. pp 1693-1705

23. Kim E, Zylstra GJ (1999) Functional analysis of genes involved in biphenyl, naphthalenes, phenanthrene, and m-xylene degradation by Sphingomonas yanoikuyae b1. J Ind Microbiol Biotechnol 23:294-302. https://doi.org/10.1038/sj.jim.2900724

24. Kosako Y, Yabuuchi E, Naka T, et al (2000) Proposal of Sphingomonadaceae fam. nov., consisting of Sphingomonas Yabuuchi et al. 1990, Erythrobacter shiba and shimidu 1982, Erythromicrobium Yurkov et al. 1994, Porphyrobacter Fuerst et al. 1993, Zymomonas Kluyver and van Niel 1936, and Sandaracinobac. Microbiol Immunol 44:563-575. https://doi.org/10.1111/j.13480421.2000.tb02535.x

25. Lagesen K, Hallin P, Rødland EA, et al (2007) RNAmmer: Consistent and rapid annotation of ribosomal RNA genes. Nucleic Acids Res 35:3100-3108. https://doi.org/10.1093/nar/gkm160

26. Leahy JG, Colwell RR (1990) Microbial degradation of hydrocarbons in the environment. Microbiol Rev 54:305-315. https://doi.org/10.1128/mmbr.54.3.305-315.1990

27. Liu X, Gai Z, Tao F, et al (2012) Carotenoids play a positive role in the degradation of heterocycles by Sphingobium yanoikuyae. PLoS One 7:. https://doi.org/10.1371/journal.pone.0039522

28. Liu X xin, Hu X, Cao Y, et al (2019) Biodegradation of Phenanthrene and Heavy Metal Removal by Acid-Tolerant Burkholderia fungorum FM-2. Front Microbiol 10:

https://doi.org/10.3389/fmicb.2019.00408

29. Lowe TM, Eddy SR (1996) TRNAscan-SE: A program for improved detection of transfer RNA genes in genomic sequence. Nucleic Acids Res 25:955-964. https://doi.org/10.1093/nar/25.5.0955

30. Marçais G, Delcher AL, Phillippy AM, et al (2018) MUMmer4: A fast and versatile genome alignment system. PLoS Comput Biol. https://doi.org/10.1371/journal.pcbi.1005944

31. Martin RG, Rosner JL (2001) The AraC transcriptional activators. Curr. Opin. Microbiol. 4:132- 137

32. Mavromatis K, Ivanova NN, Chen I min A, et al (2009) The DOE-JGI standard operating procedure for the annotations of microbial genomes. Stand Genomic Sci 1:63-67. 
https://doi.org/10.4056/sigs.632

33. Mesarch MB, Nakatsu CH, Nies L (2000) Development of catechol 2,3-dioxygenase-specific primers for monitoring bioremediation by competitive quantitative PCR. Appl Environ Microbiol 66:678-683. https://doi.org/10.1128/AEM.66.2.678-683.2000

34. Oltmanns RH, Muller R, Otto MK, Lingens F (1989) Evidence for a new pathway in the bacterial degradation of 4-fluorobenzoate. Appl Environ Microbiol 55:2499-2504. https://doi.org/10.1128/aem.55.10.2499-2504.1989

35. Pal S, Kundu A, Banerjee T Das, et al (2017) Genome analysis of crude oil degrading Franconibacter pulveris strain DJ34 revealed its genetic basis for hydrocarbon degradation and survival in oil contaminated environment. Genomics 109:374-382. https://doi.org/10.1016/j.ygeno.2017.06.002

36. Pritchard L, Glover RH, Humphris S, et al (2016) Genomics and taxonomy in diagnostics for food security: Soft-rotting enterobacterial plant pathogens. Anal Methods 8:12-24. https://doi.org/10.1039/c5ay02550h

37. Ryoo D, Shim H, Arenghi FLG, et al (2001) Tetrachloroethylene, trichloroethylene, and chlorinated phenols induce toluene-o-xylene monooxygenase activity in Pseudomonas stutzeri OX1. Appl Microbiol Biotechnol 56:545-549. https://doi.org/10.1007/s002530100675

38. Seemann T (2014) Prokka: Rapid prokaryotic genome annotation. Bioinformatics 30:2068-2069. https://doi.org/10.1093/bioinformatics/btu153

39. Takeuchi M, Hamana K, Hiraishi A (2001) Proposal of the genus Sphingomonas sensu stricto and three new genera, Sphingobium, Novosphingobium and Sphingopyxis, on the basis of phylogenetic and chemotaxonomic analyses. Int J Syst Evol Microbiol 51:1405-1417. https://doi.org/10.1099/00207713-51-4-1405

40. Tatusova T, DiCuccio M, Badretdin A, et al (2016) NCBI prokaryotic genome annotation pipeline. Nucleic Acids Res 44:6614-24. https://doi.org/10.1093/nar/gkw569

41. Top EM, Springael D (2003) The role of mobile genetic elements in bacterial adaptation to xenobiotic organic compounds. Curr Opin Biotechnol 14:262-269. https://doi.org/10.1016/S09581669(03)00066-1

42. United States National Academy of S (1983) "Polycyclic aromatic hydrocarbons: Evalutions of sources and effects." In: Committee on pyrene selected Analogues, Board on Toxicological and Environmental Health Hazards. Commission on Life Sciences \& National Research Council. p 479

43. Volkering F, Breure AM, Sterkenburg A, van Andel JG (1992) Microbial degradation of polycyclic aromatic hydrocarbons: effect of substrate availability on bacterial growth kinetics. Appl Microbiol Biotechnol 36:548-552. https://doi.org/10.1007/BF00170201

44. Wattam AR, Abraham D, Dalay O, et al (2014) PATRIC, the bacterial bioinformatics database and analysis resource. Nucleic Acids Res 42:. https://doi.org/10.1093/nar/gkt1099

45. Wences AH, Schatz MC (2015) Metassembler: Merging and optimizing de novo genome assemblies. Genome Biol 16:. https://doi.org/10.1186/s13059-015-0764-4 
46. White PA, Claxton LD (2004) Mutagens in contaminated soil: A review. Mutat. Res. - Rev. Mutat. Res. 567:227-345

47. Wilkinson SP, Grove A (2006) Ligand-responsive transcriptional regulation by members of the MarR family of winged helix proteins. Curr Issues Mol Biol 8:51-62. https://doi.org/10.21775/cimb.008.051

48. Yabuuchi E, Yano I, Oyaizu H, et al (1990) Proposals of Sphingomonas paucimobilis gen. nov. and comb. nov., Sphingomonas parapaucimobilis sp. nov., Sphingomonas yanoikuyae sp. nov., Sphingomonas adhaesiva sp. nov., Sphingomonas capsulata comb. nov., and Two Genospecies of the Genus Sphingomonas. Microbiol Immunol 34:99-119. https://doi.org/10.1111/j.13480421.1990.tb00996.x

49. Yang L, Peng M, Shah SS, Wang Q (2017) Transcriptome sequencing and comparative analysis of Piptoporus betulinus in response to birch sawdust induction. Forests 8:374. https://doi.org/10.3390/f8100374

50. Zerbino DR, Birney E (2008) Velvet: Algorithms for de novo short read assembly using de Bruijn graphs. Genome Res 18:821-829. https://doi.org/10.1101/gr.074492.107

51. (1998) VALIDATION LIST NO. 64: Validation of publication of new names and new combinations previously effectively published outside the IJSB. Int J Syst Bacteriol 48:327- 328. https://doi.org/10.1099/00207713-48-1-327

\section{Figures}




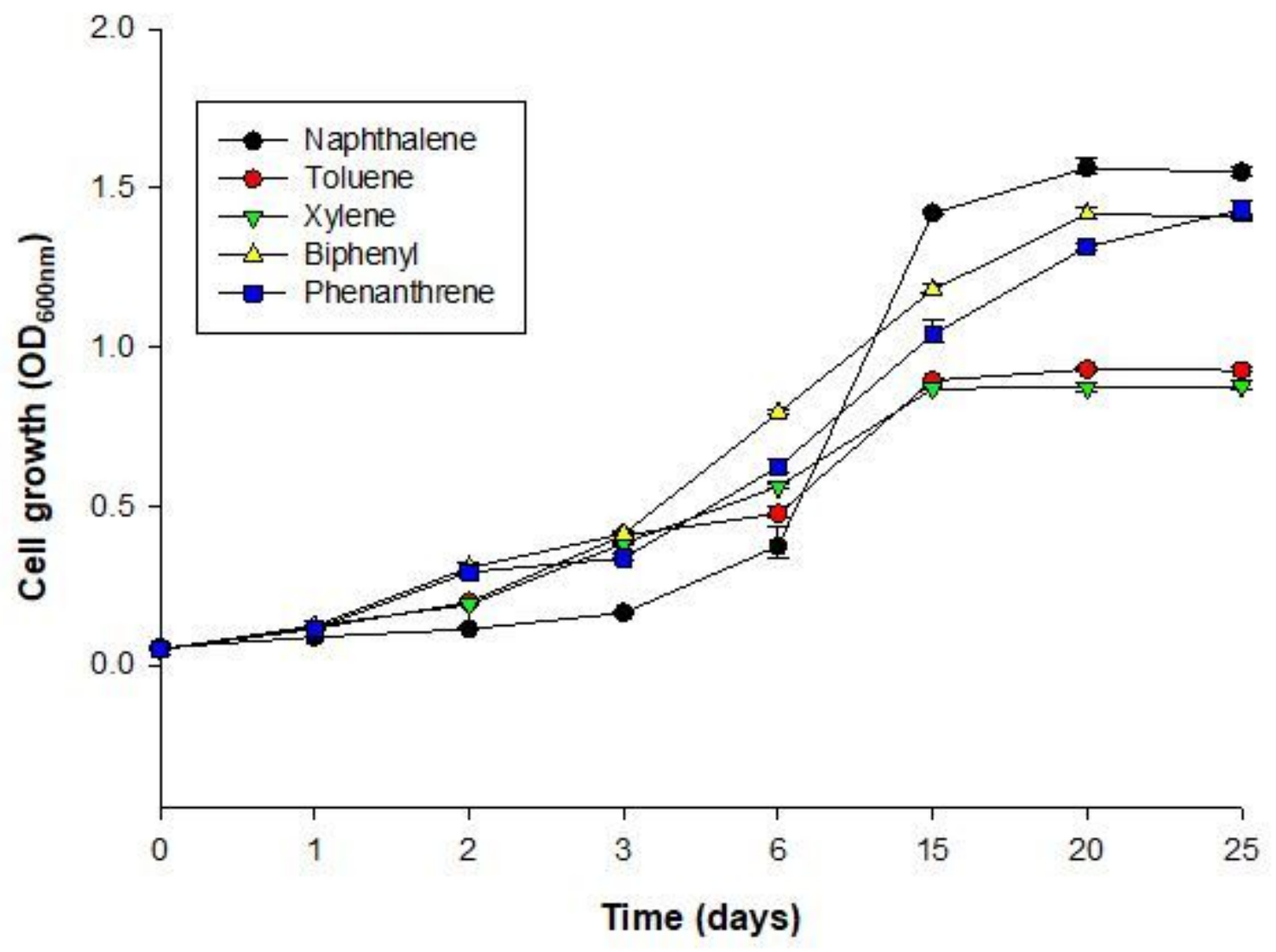

Figure 1

Growth of S. yanoikuyae S72. Bacteria were measured as optical density $(600 \mathrm{~nm})$ in liquid Bushnell Haas medium supplemented with each of the five different hydrocarbons $(100 \mu \mathrm{g} / \mathrm{mL})$ as sole source carbon for 25-day incubation. 


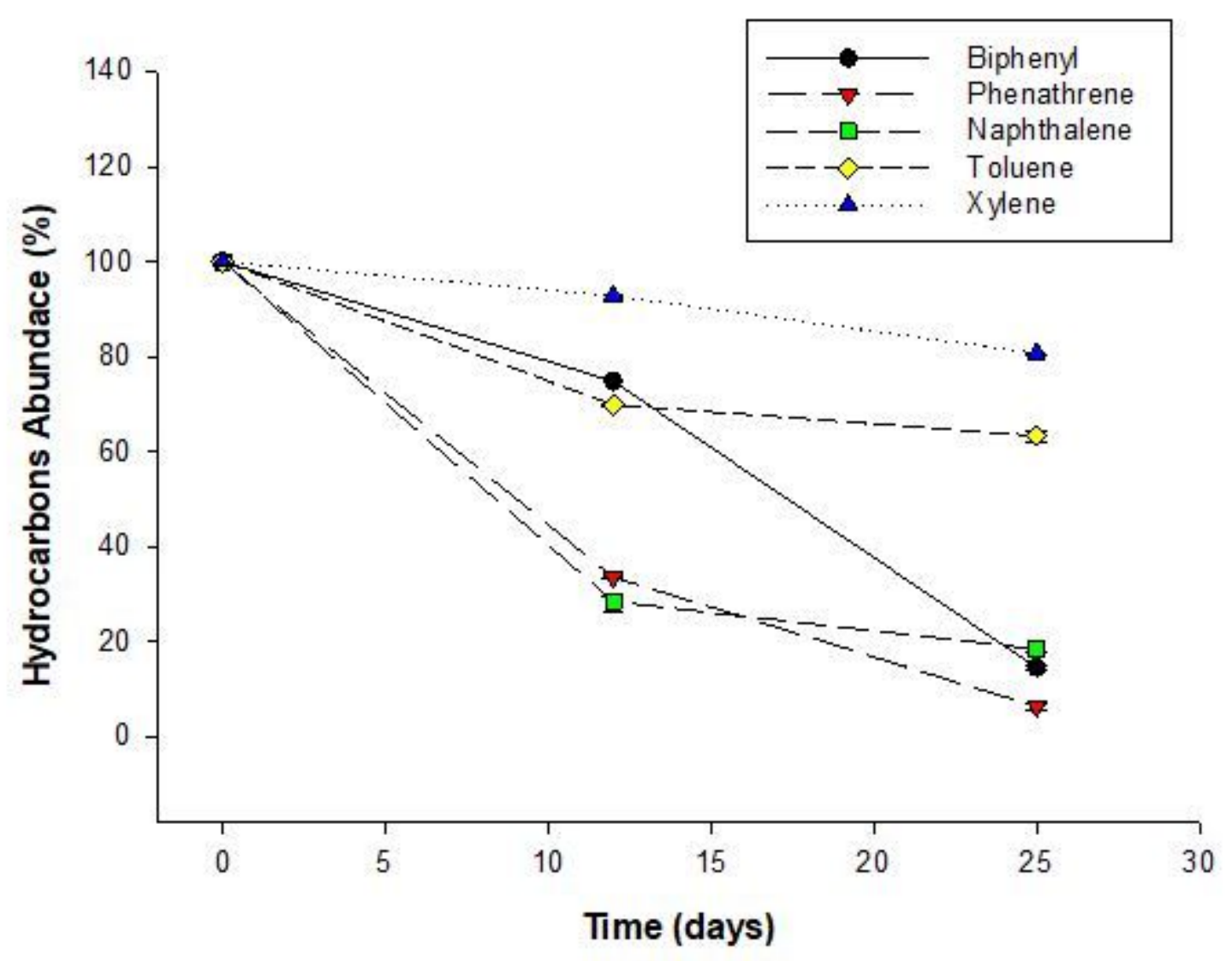

Figure 2

Degradation of hydrocarbons by S. yanoikuyae S72.. Hydrocarbon abundance (\%) of five polycyclic aromatic hydrocarbons (PAHs) in liquid Bushnell Haas medium supplemented with each of the five different hydrocarbons $(100 \mu \mathrm{g} / \mathrm{mL})$ as sole source carbon for 25 -day incubation. 


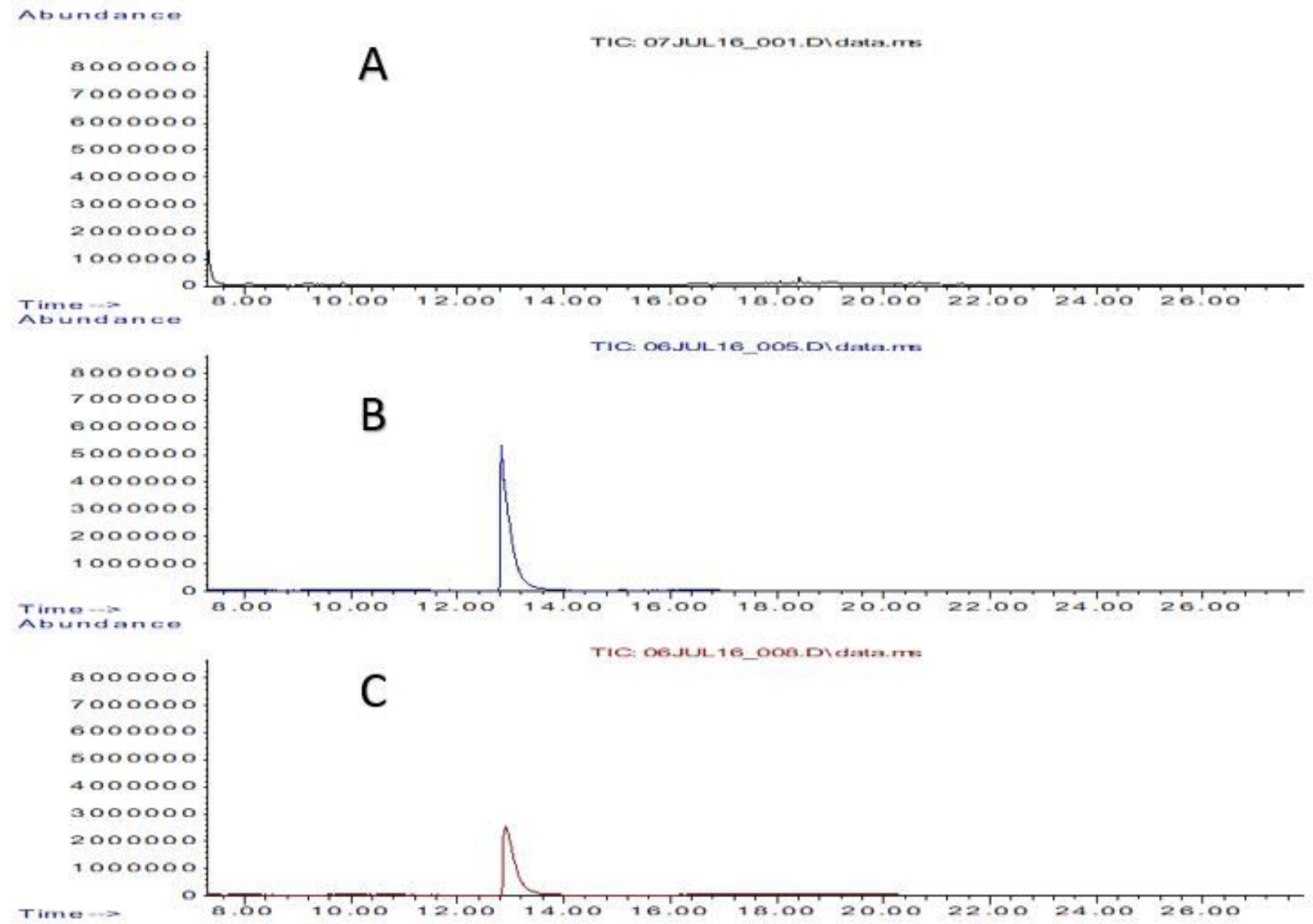

\section{Figure 3}

Gas column chromatography analysis of Naphthalene before and after degradation by Sphingobium yanoikuyae S72 A: Uninoculated culture medium without Naphthalene B: Naphthalene recovered from medium without S72 C: S72 treated naphthalene containing medium 


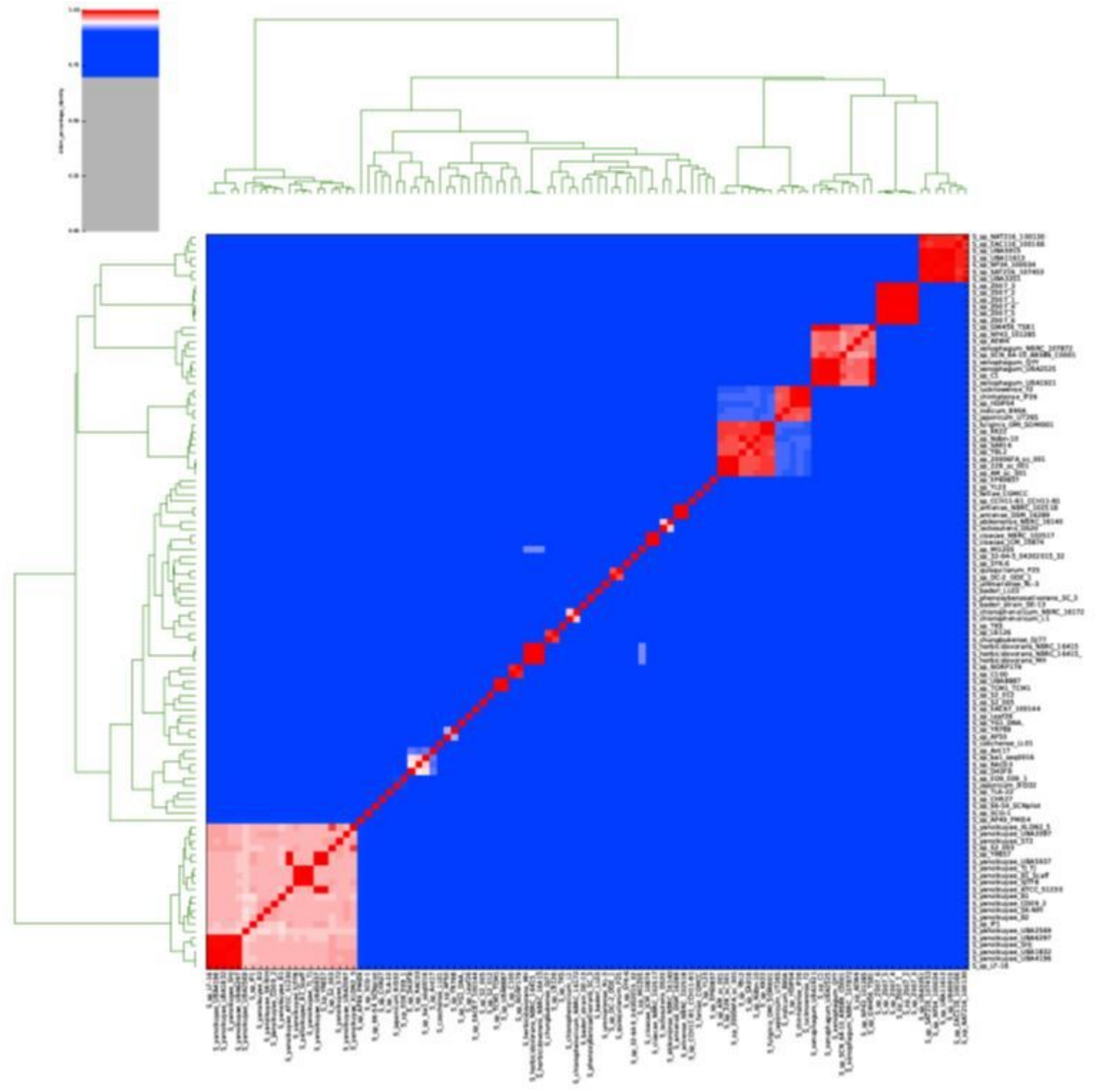

Figure 4

ANIm \% Identity of 106 Sphingobium Strains. ANI analysis based on MUMmer alignment of the genome sequences was performed using PYANI. 


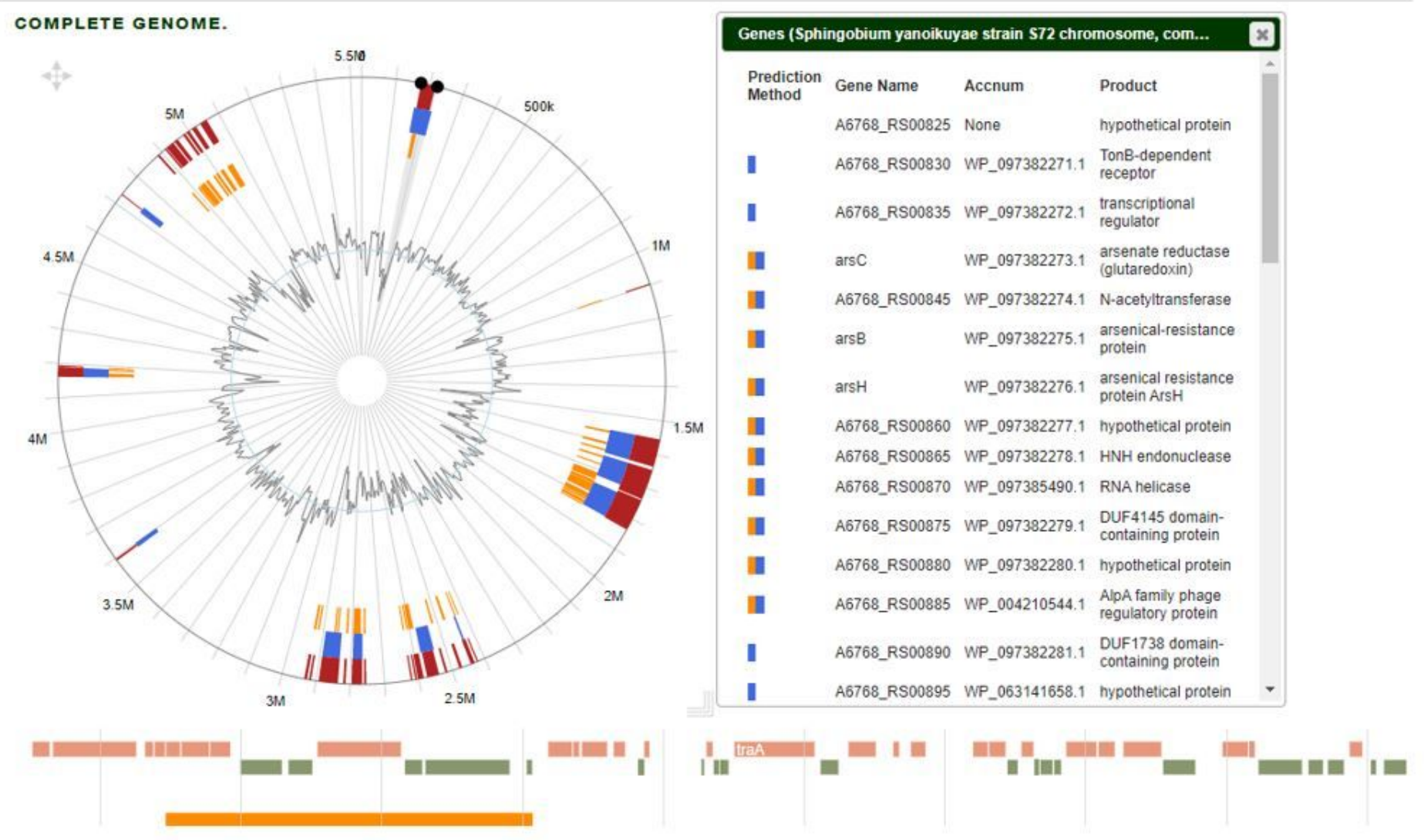

\section{Figure 5}

Genomic Island predicted in S72 with Island viewer 4

\section{Supplementary Files}

This is a list of supplementary files associated with this preprint. Click to download.

- SupplementaryfigureErickarticle.docx

- Tables1.docx 